\title{
CHARGE-TRANSFER DISTRIBUTION IN NEUTRAL CLUSTER MODELS
}

\author{
R. BAIER * and F.W. BOPP \\ Department of Theoretical Physics, University of Bielefeld, Germany
}

Received 11 February 1974

(Revised 2 May 1974)

\begin{abstract}
The charge transfer between hemispheres is investigated in the framework of a model with neutral and isotropic clusters. Without specifying the production mechanism of the clusters, the charge-transfer distribution can be uniquely determined by inclusive spectra. Minor discrepancies observed in the comparison with experimental data are attributed to the influence of the charges of the leading particles, and further tests are proposed.
\end{abstract}

\section{Introduction}

In view of recent NAL and ISR data, models with predominantly neutral and isotropically decaying clusters have received considerable attention $[1,2]$, and clustering is tentatively called the "third general feature of high energy hadron collisions" [3]. For a more detailed examination of this concept, it is useful to consider charge transfer distributions $[4,5]$, as they critically depend on the nature of the clusters.

The aim of this paper is therefore to derive relevant formulae, using the formalism of generating functionals [6] (section 2), and to discuss the presently available data $[7-10]$. We generalize the recently found relation between the dispersion of the charge-transfer distribution $[1,5]$ and the inclusive single-particle spectrum and show that independent of the mechanism of the cluster formation, all moments of the charge-transfer distribution are simply related to inclusive spectra (sect. 3 ). In such models with neutral and isotropic clusters, the charge transfer cannot better help to discriminate between special production mechanisms than just the corresponding inclusive spectra. The strong predictions of these relations are experimentally satisfied; existing minor discrepancies may be attributed to the influence of the charges of the leading particles, which is expected to vanish at ISR energies where charge independence should already hold in the central region. A suitable experiment is proposed to measure charge transfer at ISR. We derive (approximate) relations between the moments of the charge transfer distribution and inclusive spectra for fixed associated multiplicities and compare them with existing data (sect. 4).

\footnotetext{
* Supported by the Deutsche Forschungsgemeinschaft.
} 


\section{Generating functional for the neutral cluster model}

The inelastic production of particles at high energies is described in cluster models by a two-step mechanism: the formation of intermediate clusters and their subsequent decay into the observable particles $[1,2]$. For the present discussion, only one type of neutral clusters is assumed, which means that at a given mass all clusters have identical decay distributions in their rest frames. This assumption ignores differences between central and leading clusters.

A convenient way to analyse this kind of models is given in terms of generating functionals. As described in detail in a previous paper [12], the generating functional $G[\phi(q)]$ for the production of identical particles with momenta $q$ can be expressed by combining the functionals for the formation of clusters with masses $M$ and momenta $Q_{c}, G^{\mathrm{F}}\left[\phi\left(Q_{c}, M\right)\right]$, and the functional for the decay of a cluster $G^{\mathrm{D}}\left[\phi(q), Q_{c}, M\right]$ in the following way

$$
G[\phi(q)]=G^{\mathrm{F}}\left[G^{\mathrm{D}}\left[\phi(q), Q_{c}, M\right]\right],
$$

$G^{\mathrm{D}}$ with its dependence on $M$ and $Q_{c}$ is just the argument of $G^{\mathrm{F}}$. The functionals are normalized as

$$
G^{\mathrm{F}, \mathrm{D}}[\phi=1]=1 .
$$

The generalisation of eq. (1) to include charged particles (the neutrals are implicitely summed over) and neutral clusters is straight forward

$$
G\left[\phi^{+}\left(q^{+}\right), \phi^{-}\left(q^{-}\right)\right]=G^{\mathrm{F}}\left[G^{\mathrm{D}}\left[\phi^{+}\left(q^{+}\right), \phi^{-}\left(\dot{q}^{-}\right), Q_{c}, M\right]\right] .
$$

One can express the functionals in terms of the inclusive correlation functions $C_{\mathrm{K}}^{\mathrm{F}}$ for the formation of clusters and the normalized exclusive decay distributions $f_{m_{+} m_{-}}^{\mathrm{D}}$ for $m_{+}$positive and $m_{-}$negative secondaries emitted by a cluster with momentum $Q_{c}$ and mass $M$ as

$$
\begin{aligned}
& G\left[\phi^{+}\left(q^{+}\right), \phi^{-}\left(q^{-}\right)\right]=\exp \left\{\sum_{k=1}^{\infty} \frac{1}{k !} \int \prod_{i=1}^{k} \frac{\mathrm{d}^{3} Q_{c i}}{Q_{c 0 i}} \mathrm{~d} M_{i} C_{k}^{\mathrm{F}}\left(Q_{c 1}, \ldots Q_{c k}, \ldots M_{k}\right)\right. \\
& \left.\times \prod_{i=1}^{k}\left(G^{\mathrm{D}}\left[\phi^{+}\left(q^{+}\right), \phi^{-}\left(q^{-}\right), Q_{c i}, M_{i}\right]-1\right)\right\}, \\
& G^{\mathrm{D}}\left[\phi^{+}\left(q^{+}\right), \phi^{-}\left(q^{-}\right), Q_{c}, M\right]=\sum_{m_{+}, m_{-}} \frac{1}{m_{+} ! m_{-} !} \int \prod_{i=1}^{m_{+} m_{j}} \prod_{j=1} \frac{\mathrm{d}^{3} q_{i}^{+}}{q_{0 i}^{+}} \phi^{+}\left(q_{i}^{+}\right) \\
& \quad \times \frac{\mathrm{d}^{3} q_{j}^{-}}{q_{0 j}^{-}} \phi^{-}\left(q_{j}^{-}\right) f_{m_{+}, m_{-}}^{\mathrm{D}}\left(q_{1}^{+}-Q_{c}, \ldots, q_{m_{-}}^{-}-Q_{c}, M\right) .
\end{aligned}
$$


For neutral clusters, charge conservation implies an equal number of positive and negative secondaries per cluster; this restricts the summation to the values $m_{+}=m_{-}=$ $m$. For mathematical convenience, one can define spectra $\hat{f}_{m}^{\mathrm{D}}$, which are normalized to one, by

$$
\frac{1}{m_{+} ! m_{-} !} f_{m_{+}, m_{-}}^{\mathrm{D}}=\delta_{m_{+}, m_{-}} P_{m}^{\mathrm{D}}(M) \hat{f}_{m}^{\mathrm{D}}\left(q^{+}-Q_{c}, q^{-}-Q_{c}, M\right)
$$

Here $P_{m}^{\mathrm{D}}(M)$ is the probability that a cluster with mass $M$ contains $m$ charged pairs.

In the following, we only treat the dependence with respect to the cluster and particle rapidities (with the integration of the transverse momenta already performed). For the cluster decay, the following weak assumptions are made: a cluster decays isotropically in its rest frame and strong angular correlations between various momenta do not substantially destroy the isotropy as soon as one particle is produced at a fixed angle; furthermore the decay distributions are smooth around their centre. Then the rapidity dependence of $\hat{f}_{m}^{\mathrm{D}}$ is mainly determined by the Jacobian of the transformation from momenta to rapidities $[13,14]$, and $\hat{f}_{m}^{D}$ can be approximated by the product

$$
\hat{f}_{m}^{\mathrm{D}}=\prod_{i=1}^{2 m} J\left(y_{i}-y_{c}\right)
$$

and $J(y)$ is the normalized Jacobian

$$
J(y)=\frac{1}{2} \cosh ^{-2}(y)
$$

The known transverse-momentum distributions suggest that the above approximation is valid for the dominant small momenta.

Combining the last equations, the generating functional is written as

$$
\begin{aligned}
& G\left[\phi^{+}\left(y^{+}\right), \phi^{-}\left(y^{-}\right)\right]=\exp \left\{\sum_{k=1}^{\infty} \frac{1}{k !} \int \prod_{i=1}^{k} \mathrm{~d} y_{c i} \mathrm{~d} M_{i} C_{k}^{\mathrm{F}}\left(y_{c 1}, \ldots, M_{k}\right)\right. \\
& \left.\quad \times \prod_{i=1}^{k}\left(G^{\mathrm{D}}\left[\phi^{+}\left(y^{+}\right), \phi^{-}\left(y^{-}\right), y_{c i}, M_{i}\right]-1\right)\right\}
\end{aligned}
$$

with

$$
G^{\mathrm{D}}[]=\sum_{m=0}^{\infty} P_{m}^{\mathrm{D}}(M)\left[\int \mathrm{d} y^{+} \phi^{+}\left(y^{+}\right) J\left(y^{+}-y_{c}\right)\right]^{m}\left[\int \mathrm{d} y^{-} \phi^{-}\left(y^{-}\right) J\left(y^{-}-y_{c}\right)\right]^{m},
$$

which is symmetric with respect to the interchange of $\phi^{+}$and $\phi^{-}$. Functionals are used here just as a tool to obtain generating functions for discrete distributions by considering special choices of variations in the parameter functions $\phi^{+}$and $\phi^{-}$. 


\section{Charge transfer}

In order to discuss the production of charged particles in different regions, say a right hemisphere with $y>\bar{y}$ and a left one with $y<\bar{y}$, one has to substitute

$$
\phi^{ \pm}(y) \rightarrow h_{ \pm}^{\mathrm{R}} \theta(y-\bar{y})+h_{ \pm}^{\mathrm{L}} \theta(\bar{y}-y),
$$

$\theta(y)$ is the step function. Repeated differentiations of the corresponding generating function $G\left(h_{ \pm}^{\mathrm{R}}, h_{ \pm}^{\mathrm{L}}\right)$ with respect to $h_{ \pm}^{\mathrm{R}, \mathrm{L}}$ at $h_{ \pm}^{\mathrm{R}, \mathrm{L}}=0$ lead to the cross sections for producing $n_{ \pm}^{\mathrm{R}^{ \pm}}$and $n_{+}^{\mathrm{L}}$ charged particles in the considered hemispheres [15]. In this way one can derive the distribution $P^{k}(\bar{y})=\sigma^{k}(\bar{y}) / \sigma_{\text {inel }}$ in the net charge transfer $k=$ $n_{+}^{\mathrm{R}}-n_{-}^{\mathrm{R}}=n_{-}^{\mathrm{L}}-n_{+}^{\mathrm{L}}$ between these two hemispheres from a generating function $[5]$

$$
G_{\bar{y}}(x)=\sum_{k=-\infty}^{+\infty} P^{k}(\bar{y}) x^{k}
$$

which is obtained from the functional $G\left[\phi^{+}, \phi^{-}\right]$by substituting

$$
\begin{gathered}
\phi^{+}(y) \rightarrow x \theta(y-\bar{y})+\theta(\bar{y}-y), \\
\phi^{-}(y) \rightarrow \frac{1}{x} \theta(y-\bar{y})+\theta(\bar{y}-y) .
\end{gathered}
$$

The operation $(x \partial / \partial x)^{n}$ applied to $G_{\bar{y}}(x)$ at $x=1$ provides the $n$th moment of the charge-transfer probability

$$
\left\langle k^{n}(\bar{y})\right\rangle=\left.\left(x \frac{\partial}{\partial x}\right)^{n} G_{\bar{y}}(x)\right|_{x=1}=\sum_{k=-\infty}^{+\infty} k^{n} P^{k}(\bar{y}) .
$$

But unlike the multiplicity distributions, the probability $P^{k}(\bar{y})$ in eq. (11) can no longer be obtained by derivatives at $x=0$. It is therefore convenient to substitute $x=\mathrm{e}^{i t}$ and to define the characteristic function [16] $G_{\bar{y}}(t)$, which can be expressed by $P^{k}(\bar{y})$

$$
G_{\bar{y}}(t)=\sum_{k=-\infty}^{+\infty} P^{k}(\bar{y}) \mathrm{e}^{i t k}
$$

Alternative representations of this function in terms of moments $\left\langle k^{n}(\bar{y})\right\rangle$ or the equivalent cumulants $K_{n}^{\mathrm{c}}(\bar{y})$ are

$$
G_{\bar{y}}(t)=\sum_{n=0}^{\infty}\left\langle k^{n}(\bar{y})\right\rangle \frac{(i t)^{n}}{n !}=\exp \left[\sum_{n=1}^{\infty} \frac{K_{n}^{\mathrm{c}}(\bar{y})}{n !}(i t)^{n}\right] .
$$


The probability $P^{k}(\bar{y})$ can be obtained by inverting eq. (14):

$$
P^{k}(\bar{y})=\frac{1}{2 \pi} \int_{-\pi}^{+\pi} \mathrm{e}^{-i t k} G_{\bar{y}}(t) \mathrm{d} t
$$

Since for the cluster model only $G^{\mathrm{D}}\left[\phi^{+}, \phi^{-}\right]$depends explicitly on the functions $\phi^{ \pm}$, we apply the substitution in eq. (12) to $G^{\mathrm{D}}$ to derive the corresponding function $G_{\bar{y}}\left(x, y_{c}, M\right)$ for the charge transfer at $y=\bar{y}$

$$
G_{\bar{y}}^{\mathrm{D}}\left(x, y_{c}, M\right)=\sum_{m} P_{m}^{\mathrm{D}}(M)\left[1+\frac{1}{2}\left(x+\frac{1}{x}-2\right) T\left(\bar{y}-y_{c}\right)\right]^{m},
$$

where

$$
T\left(\bar{y}-y_{c}\right)=2 \int \mathrm{d} y^{+} \mathrm{d} y^{-} \theta\left(y^{+}-\bar{y}\right) \theta\left(\bar{y}-y^{-}\right) J\left(y^{+}-y_{c}\right) J\left(y^{-}-y_{c}\right)
$$

The integral over the $\cosh ^{-2}$ functions can be exactly calculated:

$$
T\left(\bar{y}-y_{c}\right)=\frac{1}{2} \cosh ^{-2}\left(\bar{y}-y_{c}\right)=J\left(\bar{y}-y_{c}\right) \text {. }
$$

One can now compare $G_{\bar{y}}^{\mathrm{D}}$ with the generating function $\widetilde{G}_{\bar{y}}^{\mathrm{D}}$ for the emission of negative (= positive) particles from a given cluster with rapidities all at $y=\bar{y} . \widetilde{G}_{\bar{y}}^{\mathrm{D}}$ is obtained by substituting

$$
\phi^{-}(y) \rightarrow 1+(z-1) \delta(y-\bar{y}), \quad \phi^{+}(y) \rightarrow 1
$$

in eq. (9), and has the explicit form

$$
\widetilde{G}_{\bar{y}}^{\mathrm{D}}\left(z, y_{c}, M\right)=\sum_{m} P_{m}^{\mathrm{D}}(M)\left[1+(z-1) J\left(\bar{y}-y_{c}\right)\right]^{m}
$$

By comparison, one observes that

$$
G \frac{\mathrm{D}}{y}\left(x=\mathrm{e}^{i t}, y_{c}, M\right)=\widetilde{G}_{\bar{y}}^{\mathrm{D}}\left(z=\frac{1}{2}\left(x+\frac{1}{x}\right)=\cos t, y_{c}, M\right) .
$$

Using eq. (8), we get the same relation for the corresponding generating functions of the overall process independent of the mechanism of the cluster formation. One can now express $\widetilde{G}_{\bar{y}}(z)$ in terms of the normalized inclusive distribution functions $\rho_{\mathrm{n}}$ for the production of negative (= positive) particles, all of them with rapidities $\bar{y}$, and obtain a representation of $G_{\bar{y}}(x)$ in terms of these spectra,

$$
G_{\bar{y}}\left(x=\mathrm{e}^{i t}\right)=\widetilde{G}_{\bar{y}}\left(\frac{x+1 / x}{2}=\cos t\right) \doteq 1+\sum_{n=1}^{\infty} \frac{(\cos t-1)^{n}}{n !} \rho_{n}^{-}(\bar{y}, \bar{y}, \ldots)
$$


We expand $(\cos t-1)^{n}$ as

$$
(\cos t-1)^{n}=n ! \sum_{m=n}^{\infty} \frac{(i t)^{2 m}}{(2 m) !} Z_{m}^{(n)},
$$

where

$$
n ! Z_{m}^{(n)}=\sum_{\substack{\left\{\nu_{1}, \ldots \nu_{n}\right\} \\ \Sigma \nu_{i}=m, \nu_{i}>0}} \frac{(2 m) !}{\left(2 \nu_{1}\right) ! \ldots\left(2 \nu_{n}\right) !}
$$

By comparing powers in $t$ in the above expression of the characteristic function with that in eq. (15), all odd moments are seen to vanish, and for the even ones the following relation is obtained:

$$
\left\langle k^{2 n}(\bar{y})\right\rangle=\sum_{m=1}^{n} Z_{n}^{(m)} \rho_{m}^{-}(\bar{y}, \bar{y}, \ldots \bar{y})
$$

Alternatively, one can describe $\widetilde{G}_{\bar{y}}$ in terms of the correlation functions $C_{n}$ for negative (or positive) secondaries to get

$$
G_{\bar{y}}\left(x=\mathrm{e}^{i t}\right)=\widetilde{G}_{\bar{y}}\left(\frac{x+1 / x}{2}=\cos t\right)=\exp \left[\sum_{n=1}^{\infty} \frac{(\cos t-1)^{n}}{n !} C_{n}^{-}(\bar{y}, \ldots, \bar{y})\right]
$$

In much the same way as before, we compare this expression with the one for the characteristic function, this time in terms of cumulants [eq. (15)]. All odd cumulants vanish; the even ones are given by

$$
K_{2 n}^{\mathrm{c}}(\bar{y})=\sum_{m=1}^{n} Z_{n}^{(m)} C_{m}^{-}(\bar{y}, \ldots, \bar{y})
$$

Explicitly, the first few relations are

$$
\begin{aligned}
& K_{2}^{\mathrm{c}}(\bar{y})=\left\langle k^{2}(\bar{y})\right\rangle=\rho^{-}(\bar{y})=\rho^{+}(\bar{y}), \\
& K_{4}^{\mathrm{c}}(\bar{y})=\left\langle k^{4}(\bar{y})\right\rangle-3\left(\left\langle k^{2}(\bar{y})\right\rangle^{2}=\rho^{-}(\bar{y})+3 C_{2}^{-}(\bar{y}, \bar{y}),\right. \\
& K_{6}^{\mathrm{c}}(\bar{y})=\rho^{-}(\bar{y})+15 C_{2}^{-}(\bar{y}, \bar{y})+15 C_{3}^{-}(\bar{y}, \bar{y}, \bar{y}) .
\end{aligned}
$$

One has to note that the input of assumptions needed to apply the described formalism can be actually somewhat relixed. The dominance of the Jacobian is not nec- 
essary if the mass dependence of the cluster decay distribution factorizes and the spectra exhibit weak factorization properties. One can again go through the steps above, with $J\left(y-y_{c}\right)$ now just an arbitrary function. Only eq. (19) will not longer be valid, but if $J(y-\bar{y})$ and hence also $T(y-\bar{y})$ are still sharply peaked (relative to the smooth formation spectrum) one can approximate

$$
T\left(\bar{y}-y_{c}\right)=\delta J\left(\bar{y}-y_{c}\right)
$$

where $\delta$ is an additional parameter depending on the properties of $J$; e.g. for the Gaussian form

$$
J(y)=\frac{1}{\sqrt{2 \pi \sigma^{2}}} \exp \left(-\frac{y^{2}}{2 \sigma^{2}}\right),
$$

a calculation gives $\delta=\sqrt{\frac{1}{2}} \pi \sigma=1.25 \sigma$ (which is almost one for the width $\sigma=0.7-$ $0.9[13,14]$. The modification due to eq. (30) leads then simply to the replacement $Z_{n}^{(m)} \rightarrow \delta^{m} Z_{n}^{(m)}$ in the results given in eqs. (26) and (28).

\section{Comparison with experimental data}

Concerning the relations between the moments of the charge transfer distribution and the inclusive charged particle distributions, it is important to point out that they hold irrespective of the production mechanism of the clusters; the only essential assumptions are the neutrality and isotropy of the clusters. The moments of the chargetransfer distribution therefore cannot better distinguish between various formation mechanism than the corresponding inclusive spectra. This means, for instance, that the dispersion $\left\langle k^{2}(\bar{y})\right\rangle$ cannot discriminate between suitable multiperipheral-, independent emission-, or even nova-(fragmentation-) type cluster models, at least as long as all these models are dealing with predominantly neutral, almost isotropically decaying clusters. Nevertheless measurements of charge transfer quantities in addition to inclusive distributions are very helpful in order to test the cluster concept and the above stated assumptions.

Fig. 1 shows the experimental values of the dispersion $[7-10]\left\langle k^{2}(0)\right\rangle$ and the corresponding points of the inclusive single-particle spectra $[10,17]$ for positive and negative pions $\left(\rho^{+}(0)\right.$ and $\left.\rho^{-}(0)\right)$ for proton-proton collisions. According to the model, all the points should be equal at fixed energy, but one finds that the equality [eq. (29a)] is violated for $\rho^{-}(0)$ by about $50 \%$ at $p_{\text {lab }}=12 \mathrm{GeV} / c$ and by $30 \%$ at 102 $\mathrm{GeV} / c$, whereas the agreement between the dispersion and $\rho^{+}(0)$ is much better. Furthermore, one observes that the considered quantities approach each other with increasing energy. It seems therefore quite probable that the compared values will coincide at energies for which $\rho^{+}(0) \simeq \rho^{-}(0)$. The observed discrepancy can just be attributed to the additional positive charge coming from the leading proton (via $p \rightarrow n+$ $\left.\mathrm{x}_{\text {slow }}^{+}\right)$. Such charges obviously contribute to the single-particle spectrum $\rho^{+}(0)$ and 


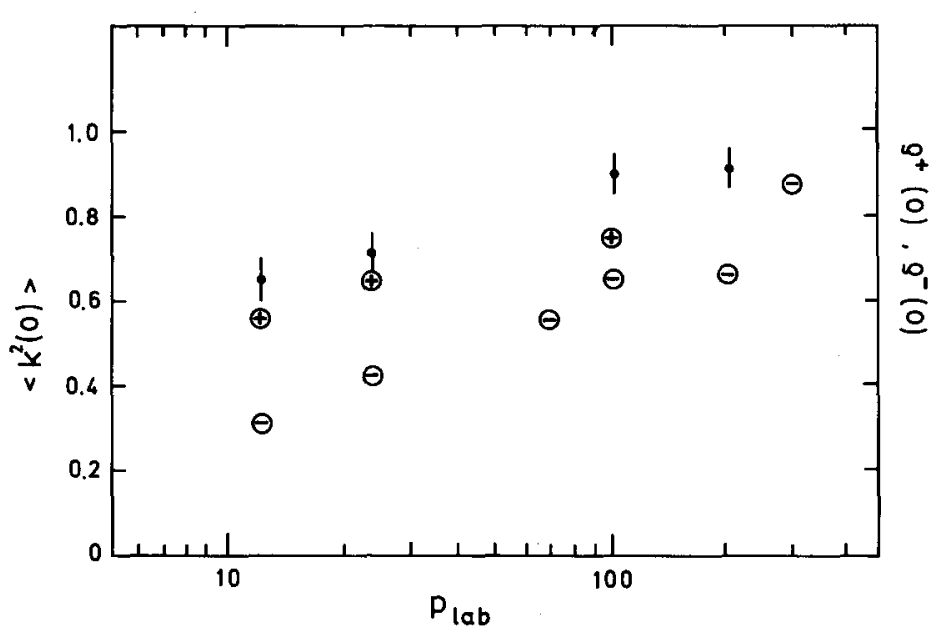

Fig. 1. Data on the dispersion $\left\langle k^{2}(0)\right\rangle$ and on inclusive single particle spectra for positive $(\oplus)$ and negative $(\theta)$ pions in inelastic proton-proton collisions $[7-10,17]$.

to the charge-transfer distribution ${ }^{\dagger}$.

A more conclusive test can be expected at ISR energies, where the charge distribution $\mathrm{d} Q / \mathrm{d} y$ should be negligibly small in the central region. Instead of measuring the charge transfer between hemispheres, for which the whole phase space has to be covered, it would be interesting to measure the distribution with respect to the difference of positive and negative charges in a fixed in terval $[-\bar{y}, \bar{y}]$ around the centre. This interval has to be small enough so that its boundaries lie within the central region, but large enough so that the decay products of one cluster do not contribute significantly to the charge transfer at both of the boundary points. A value of $\bar{y}=1.2$ will be a reasonable choice in order to fulfill these conditions. The cluster model then predicts, within an estimated error of about $1 \%$, that the dispersion of this distribution should be equal to the sum of the single-particle spectra at $\bar{y}$ and $-\bar{y}$, respectively,

$$
\left\langle k^{2}(-\bar{y}, \bar{y})\right\rangle=\rho(-\bar{y})+\rho(\bar{y}), \quad \bar{y}=1.2
$$

if charge independence already holds for the spectra, $\rho^{+}=\rho^{-}$.

According to eqs. (26) and (28), the higher moments of the charge-transfer distribution are related to the higher correlation functions. With the existing protonproton data at 102 and $205 \mathrm{GeV} / c[10]$, one can compare $\left\langle k^{4}(0)\right\rangle$ with the value of

$\dagger$ An estimate of this additional charge transfer in a multi-Regge model is $\left\langle k^{2}(0)\right\rangle$ leading charge $=$ $\left(2 \alpha_{p}^{\text {eff }}-2 \alpha_{R}^{\text {eff }}\right)^{-1}\left(\rho^{+}(0)-\rho^{-}(0)\right)$. 


$$
\rho(0)[1+3 \rho(0)(R(0,0)+1)] \simeq 2.3-3.0,
$$

using $R^{--}(0,0) \simeq R^{++}(0,0) \simeq 0.35-0.40$ and $\rho^{-}(0)=0.65$ or $\rho^{+}(0)=0.75$, respectively. Since $\left\langle k^{4}(0)\right\rangle$ is even more sensitive than the dispersion to the higher chargetransfer cross sections with larger errors the calculated value of $\left\langle k^{4}(0)\right\rangle=2.5-3.0$ has to be taken with caution.

A further test of the cluster-model approach to inelastic scattering can be found by relating the following moments of the charge-transfer distribution

$$
\left\langle k^{2 m}(\bar{y})\right\rangle_{n}=\sum_{k=-n}^{n} k^{2 m} \frac{\sigma_{n}^{k}(\bar{y})}{\sigma_{\text {inel }}}
$$

to the $l$-particle spectrum $\rho_{n}^{l}\left(y_{1}, \ldots y_{l}\right)$, both with fixed associated multiplicity $n\left(n=n_{+}=n_{-}\right)$. If this multiplicity is not too small to destroy the assumed factorization properties, one can apply the arguments given above to derive

$$
\left\langle k^{2 m}(\bar{y})\right\rangle_{n}=\sum_{l=1}^{m} Z_{m}^{(l)} \rho_{n}^{l}(\bar{y}, \ldots \bar{y}), \quad \rho_{n}^{l}=0 \text { for } n<l .
$$

A comparison of the available data $[7-10,17]$ - here mainly just indirectly obtained from figures - for dispersion $\left\langle k^{2}(0)\right\rangle_{n}$ and the distribution

$$
\rho_{n}^{1}(0)=\left.\frac{1}{\sigma_{\text {inel }}} \frac{\mathrm{d} \sigma_{n}}{\mathrm{~d} y}\right|_{y=0}
$$

for positive and negative pions is given in fig. 2 for various multiplcities (instead of the normalization to the inelastic cross section the normalisation to the corresponding prong cross sections [18] is used). It can be seen that the discrepancies found between $\left\langle k^{2}(0)\right\rangle$ and $\rho^{+}(0)\left(\rho^{-}(0)\right)$ seem to show up mainly at the four-prong level and that they decrease with increasing associated multiplcity. This may be an additional indication that effects due to the charges of the leading particles - which should for statistical reasons show up dominantly at lower multiplicities - are indeed responsible for the above disagreement. Some clarification of this picture might come from a measurement of the rapidity dependence of the dispersion $\left\langle k^{2}(\bar{y})\right\rangle_{n}$ for fixed associated multiplicities.

According to eq. (28), one can determine the cumulants $K_{2 n}^{c}(\bar{y})$ from the correlation functions $C_{l}(\bar{y}, \bar{y} \ldots)$. Therefore the energy dependence of the charge-transfer probability $P^{k}(\bar{y})$ is related to that of the correlation functions. For the charge-transfer distribution from forward to backward hemispheres, $\bar{y}=0$, we have

$$
P^{k}(0)=\frac{1}{2 \pi} \int_{-\pi}^{+\pi} \mathrm{d} t \mathrm{e}^{-i t k} \exp \left\{\sum_{n=1}^{\infty} \frac{(i t)^{2 n}}{(2 n) !} \sum_{l=1}^{n} Z_{n}^{(l)} C_{l}(0,0, \ldots 0)\right\} .
$$




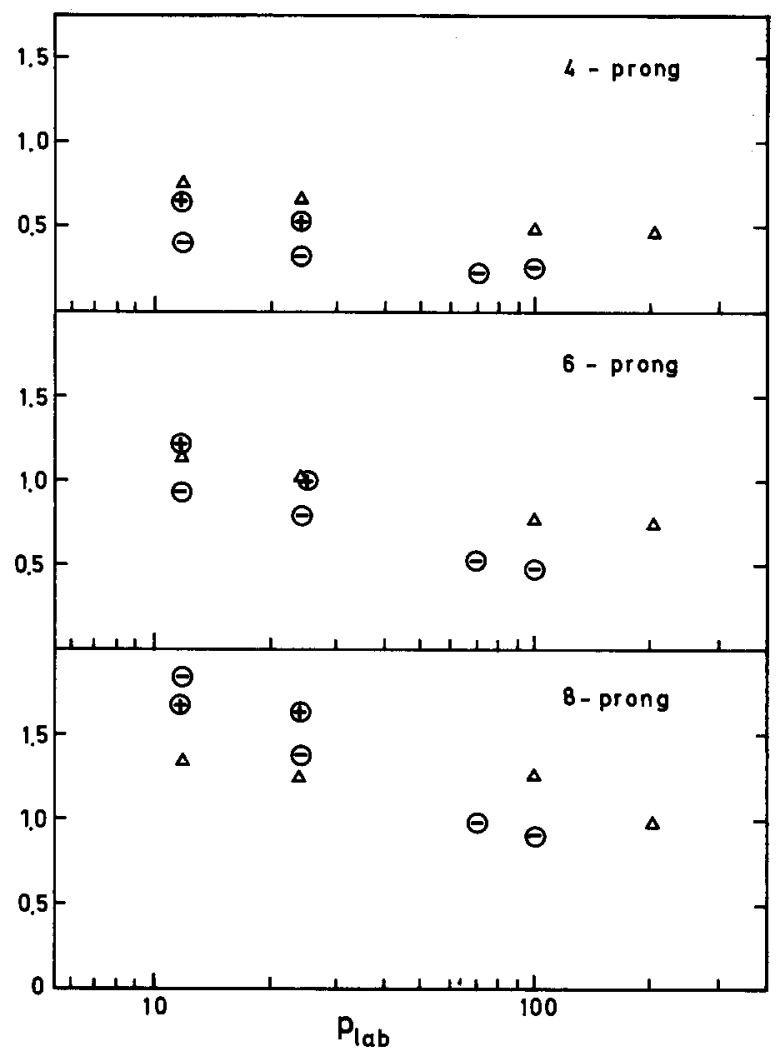

Fig. 2. Data on the normalized dispersion $\Sigma k^{2} \sigma_{n}^{k} / \sigma_{n}$ and the distribution $\left(1 / \sigma_{n}\right) / \mathrm{d} \sigma_{n}(y=0) / \mathrm{d} y$ for positive $(\Theta)$ and negative $(\Theta)$ pions for different associated multiplicities in inelastic protonproton collisions $[7,10,17,18]$.

Because of the weak energy dependence observed for $\rho^{\mathrm{ch}}(0)$ and $R^{\mathrm{ch}}(0,0)$ at NAL and ISR energies, a weak dependence of $P^{k}(0)$ on energy is indicated as it is indead observed.

It is now interesting to approximate the integral [eq. (35)] by assuming that the correlation functions for equally charged particles at the centre, $C_{l}(0,0, \ldots)$, decrease strongly with increasing order $l$. Such a behaviour is to be expected in the neutral cluster model, if the average number of particles per cluster is small. An indication may be found from existing data, when one compares $C_{1}^{-}(0)=\rho^{-}(0) \simeq 0.65$ with $C_{2}^{-}(0,0) \simeq 0.17$ and $\rho^{+}(0) \simeq 0.75$ with $C_{2}^{+}(0,0) \simeq 0.22$ at NAL energies [10] (there is of course a strong dependence on the size of the diffractive component). In this approach, the integral can now be performed:

$$
P^{k}(0)=\mathrm{e}^{-\frac{1}{2} C_{2}(0,0)-z} \sum_{l=0}^{\infty} \frac{1}{l !}\left(\frac{C_{2}(0,0)}{2}\right)^{l}\left(\frac{\partial}{\partial z}\right)^{2 l} I_{k}(z)+\mathrm{O}\left(C_{3}\right)
$$


where $I_{k}(z)$ are the modified Bessel functions [19] and $z=\rho(0)-C_{2}(0,0)$. At NAL energies, only the first two terms of the sum contribute significantly, and they give for the charge-transfer cross sections $\sigma^{k=0} \simeq 17 \mathrm{mb}, \sigma^{|k|=1} \simeq 12 \mathrm{mb}$ and $\sigma^{|k|=2} \simeq$ $3 \mathrm{mb}$, which have to be compared with the measured values of $15.8 \pm 2.7(15.6 \pm 3.0)$, $13.0 \pm 2.7(13.3 \pm 3.0)$ and $2.6 \pm 0.5(3.2 \pm 0.7)$ at $P_{\mathrm{lab}}=102 \mathrm{GeV} / c(205 \mathrm{GeV} / c)$

\section{References}

[1] A. Biatas, CERN reprint TH 1745 (1973), and references therein.

[2] E.L. Berger, CERN preprint TH 1737 (1973).

[3] S. Pokorski and L. Van Hove, CERN preprint TH 1772 (1973).

[4] T.T. Chou and C.N. Yang, Phys. Rev. D7 (1973) 1425;

K. Fialkowski, MPI preprint (1973);

D.R. Snider, Phys. Rev. D7 (1973) 3517;

Ph. W. Coulter and D.R. Snider, preprint ANL/HEP 7340 (1973).

[5] C. Quigg and G.H. Thomas, Phys. Rev. D7 (1973) 2752.

[6] L.S. Brown, Phys. Rev. D5 (1972) 748;

S.-S. Shei and T.-M. Yan, Phys. Rev. D6 (1972) 1744.

[7] U. Idschok et al., Munich preprint, MPI-PAE/Exp. E. 1.29 (1973).

[8] C. Bromberg et al., preprint UMBC 73-19/UR-460 (1973);

D. Cohen, preprint COO-3065-60, UR-461 (1973).

[9] S. Barish et al., NAL-Conf.-73/25-Exp (1973).

[10] J.C. Vander Velde, Recent results from the NAL bubble chamber, 5th Int. Conf. on high energy collisions, Stony Brook, 1973.

[11] P. Slattery, Phys. Rev. D7 (1973) 2073.

[12] F.W. Bopp, Nucl. Phys. B68 (1974) 551.

[13] E.L. Berger and A. Krzywicki, Phys. Letters 36B (1971) 380;

E.L. Berger, G.C. Fox and A. Krzywicki, Phys. Letters 43B (1973) 132.

[14] A. Bialas, K. Fiakkowski and K. Zalewski, Phys. Letters 45B (1973) 337.

[15] D. Sivers and G.H. Thomas, preprint ANL/HEP 7328, SLAC-PUB 1308 (T/E) (1973).

[16] H. Cramér, Mathematical methods of statistics (Princeton University Press, 1946).

[17] V. Blobel et al., DESY preprint 73/36 (1973);

E. Pauli, 2nd Int. Conf. on elementary particles, Aix-en-Provence Proc., p. Cl-369 (1973).

[18] V.V. Ammosov et al., Phys. Letters 42B (1972) 519;

C. Bromberg et al., Phys. Rev. Letters 31 (1973) 1563;

G. Charlton et al., Phys. Rev. Letters 29 (1972) 515.

[19] M. Abramowitz and I. Stegun, Handbook of mathematical functions, (Dover Publ., N.Y., 1965). 